\title{
SENSORY, MOTOR, AND REFLEX CHANGES IN TWO CASES OF INTRACTABLE PAIN AFTER STEREOTACTIC MESENCEPHALIC TRACTOTOMY
}

\author{
BY
}

ANSGAR TORVIK

\section{From the Neurosurgical Clinic, Lund, Sweden, and the Department of Pathology, Ullevål Hospital, Oslo, Norway}

Section of the spino- and trigeminothalamic fibres at the level of the mesencephalon for intractable pain was first performed by Dogliotti (1938). A few years later Walker (1942a) developed a standard technique for section of the pain fibres at the level of the posterior margin of the superior colliculus, and later several cases of mesencephalic tractotomy were published (Guiot and Forjaz, 1947; David, Talairach, and Hécaen, 1947; Sjöquist, 1949; Walker, 1950; Schwartz, 1950; White and Sweet, 1955). Since this procedure involves a somewhat high mortality, attempts have been made at the Neurosurgical Clinic in Lund to destroy the spinothalamic tract at mesencephalic levels by stereotactic methods. So far the operation has been tried in only a few cases. The present report represents an analysis of the neurological and necropsy findings in two patients.

Both cases presented were operated upon by Professor Leksell in Lund. The technique and apparatus employed have been described elsewhere (Leksell, 1957), and will only be briefly commented upon below.

\section{Case Reports}

Case 1.-The patient was a 19-year-old girl suffering from cancer in the right lung. Her pulmonary symptoms started in June, 1956, and at the time the diagnosis was confirmed the tumour was considered inoperable. She was admitted to the Neurosurgical Clinic in Lund, Sweden, on December 14, 1956, because of unbearable pain in the right upper extremity and the right half of the chest. The pain was considered to be due to metastases with pressure on the right brachial plexus and intercostal nerves, and it was decided to perform a left-sided mesencephalic tractotomy.

Pre-operative Neurological Examination.-In addition to the spontaneous pain, she complained of intense cutaneous hyperaesthesia in the right upper extremity and right side of the chest. There was a slight hypoaesthesia to pinprick within the same area, but no sensory loss in the rest of the body. Muscle-joint sensation and stereognosis were normal. The right upper extremity showed some diffuse muscular atrophy and was somewhat weaker than the left. These symptoms were probably in part due to inactivity caused by the hyperaesthesia. There were no pareses of the other extremities, and movements were rapidly and precisely performed in all limbs. The abdominal, plantar, and tendon reflexes were normal. Examination of the cranial nerves did not reveal any abnormalities.

Operation.-The operation was performed under local anaesthesia on December 21, 1956. The stereotactic apparatus developed by Leksell (1957) was used, and the lesion was produced by bipolar thermocoagulation to $52^{\circ} \mathrm{C}$. The electrodes were introduced through a small burr hole in the left parieto-occipital region with the mesencephalic aqueduct and posterior commissure as reference points. Stimulation of both electrodes before the coagulation produced a sensation of pain in the entire right half of the body, and the operation resulted in subtotal analgesia in the right half of the body. No detailed neurological examination was made immediately after the operation.

Post-operative Course.- The patient recovered from the operation within a few days. Her general condition improved considerably, and she got out of bed and started walking a little. She still felt slight pain in the right side of the chest and arm, but it was insignificant compared with the pre-operative state, and the dose of analgesics was reduced to a minimum. However, six weeks after the operation she again gradually got worse, with increasing dyspnoea and emaciation. She now had severe pain in the left side of the chest, and gradually also in the right side. There was no certain increase of the pain in the right arm, and the pain in the right side of the chest was considerably less than on the left. The dose of analgesics again reached the pre-operative level. During the last weeks it was difficult to obtain reliable information concerning the intensity of the pain on the two sides. She died from increasing respiratory distress and general emaciation on March 9, 1957, two and a half months after the operation.

Post-operative Neurological Examinations.-The first complete neurological examination was performed four days after the operation. She was then analgesic to pinprick on the right arm and leg (Fig. 1) but strong pinching of the skin evoked a diffuse disagreeable feeling 


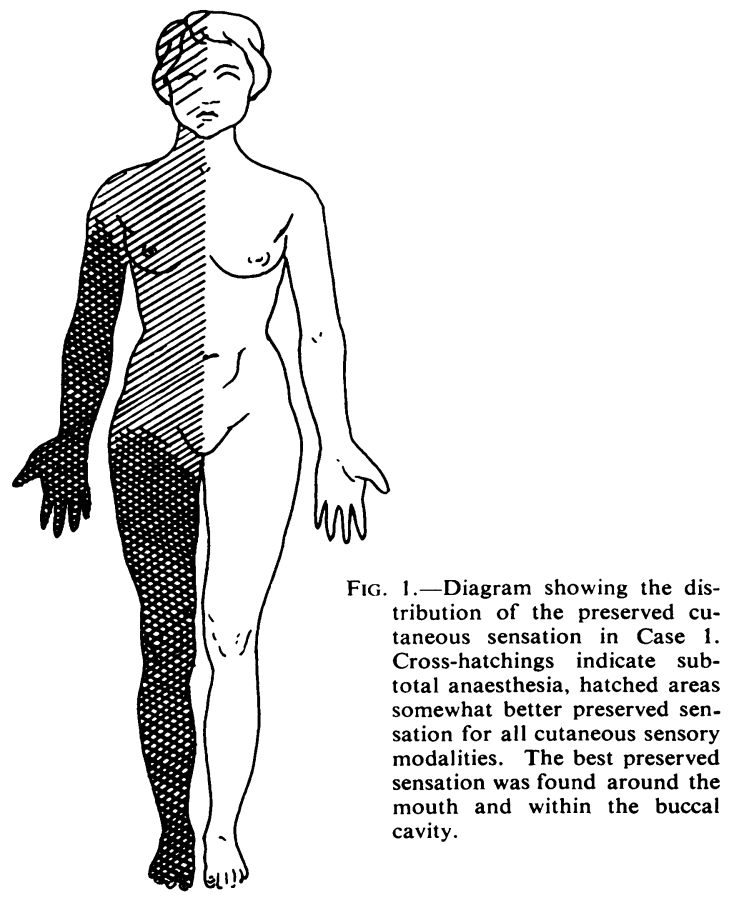

which spread to most of the extremity. The same paraesthesiae were produced by vibration from a tuning fork. In the right half of the trunk there was a slight, but distinct pain reaction to pinprick, which was fairly well localized. The same degree of hypoalgesia was found in the right half of the forehead, while the sensation was somewhat better preserved on the chin. Around the mouth and lips and in the right half of the buccal cavity and tongue, there was a rather strong reaction to pain precisely localized. The best preserved pain sensitivity was found within the mouth. A similar distribution was found for preserved temperature and toc ch. Strong warm and cold stimuli were seldom correctly perceived on the right arm and leg, while temperature differences were rather wel! differentiated on the trunk and particularly in and around the mouth. Touch stimuli were frequently felt on the right arm and leg, but very poorly localized. On the trunk light touch stimuli were perceived and more precisely localized while the best preservation was found within the buccal cavity (Fig. 1). Muscle-joint sensitivity was very poor. She could sometimes localize movements in the shoulder, knee, etc., but could not tell which finger or toe was moved. The taste sensation was greatly diminished on the right half of the tongue, but not abolished. On the left side of the body all sensory qualities were normal.

During the further course there was no change in the sensory status except for some increase in the paraesthesiae on pinching the skin. They could, however, only be produced by rather intense stimuli.

From the first day after operation it was noted that the right plantar response was extensor. On examination four days after the operation there was a slight paresis of the right arm and leg. The tendon reflexes were considerably increased on the right side with clonic ankle jerk, and there was possibly also a slight increase of the left patellar and Achilles reflexes. The right abdominal reflexes were almost abolished. During the following days the tendon reflexes on the right side grew strongly hyperactive, and there also appeared to be some increase of the left knee and ankle jerks. The strength of the right arm and leg gradually improved. One month after the operation the strength of the right arm was definitely better than pre-operatively, and there was practically no paresis of the right leg. Because of the loss of deep sensibility, finer tests for motor performance could not be made. There was no spasticity, and the strength and movements of the left extremities were normal. From the first month after the operation until death no further changes in the motor or reflex findings were observed.

Necropsy.-The whole right lung was infiltrated by a bronchial carcinoma which spread across the mediastinum and compressed the left main bronchus. Metastases were found. in regional lymph nodes, the chest wall, and the right supraclavicular fossa.

The brain was fixed in situ by intra-arterial formalin perfusion. The spinal canal was not opened, and only the brain and the upper cervical segments of the spinal cord were removed. After further fixation in $96 \%$ alcohol the brain was cut and carefully examined. There were no signs of metastases. Nor was there any bleeding of importance along the electrode tracks. The brain-stem was cut in $15-20 \mu$ thick transverse serial sections, and every tenth section was mounted and stained with thionin to determine the extent of the lesion and for further anatomical studies. Other sections were

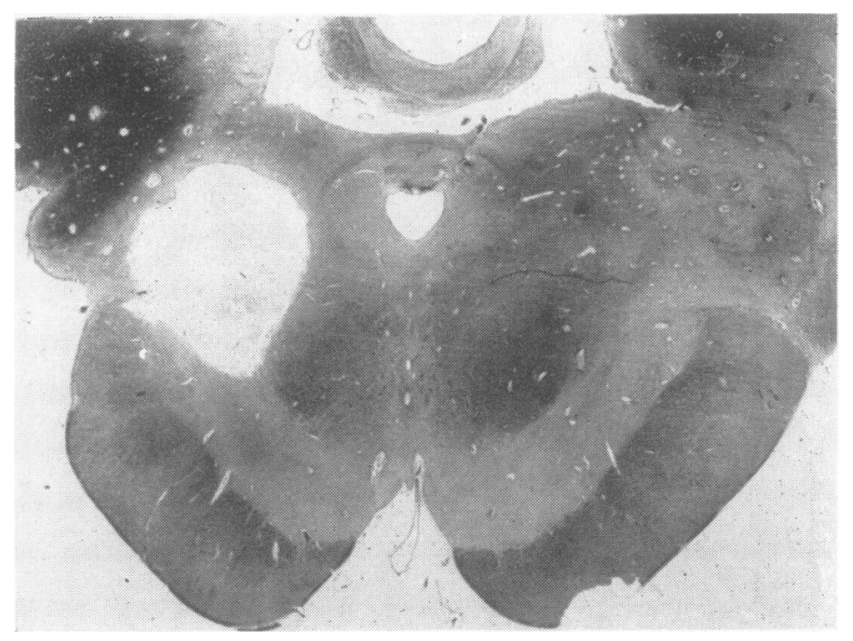

FIG. 2.-Photomicrograph of a Weil-stained section at the level of the lesion in Case 1. 


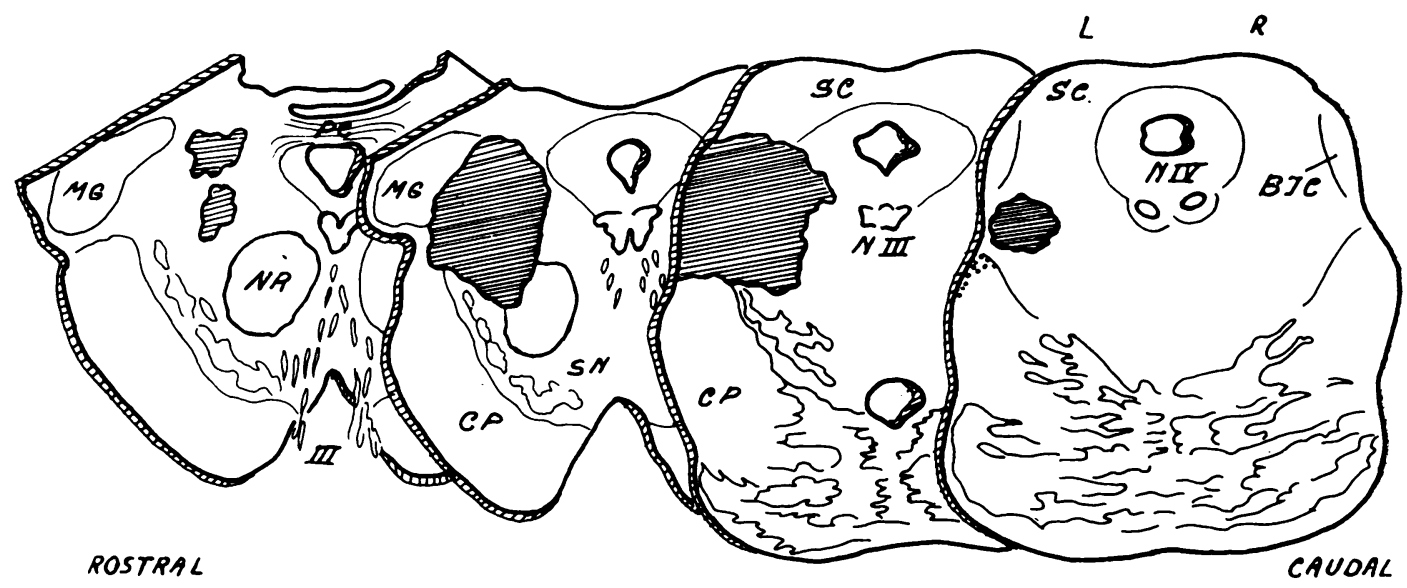

FIG. 3.-Drawings made by means of a projection apparatus showing the extent of the lesion at various levels in Case 1. The stippled area in the drawing to the right shows the localization of the degenerated fibres in the cerebral peduncle.

\author{
BIC : Brachium of inferior colliculus. \\ $\mathrm{CM}$ : Centrum medianum. \\ CP : Cerebral peduncle (basis pedunculi) \\ FMT : Mamillothalamic fasciculus. \\ L: $\quad$ Left \\ MG : Medial geniculate body. \\ NH : Habenular nuclei. \\ NM : Nucleus dorsomedialis of thalamus. \\ NP : Nuclei of pulvinar.
}

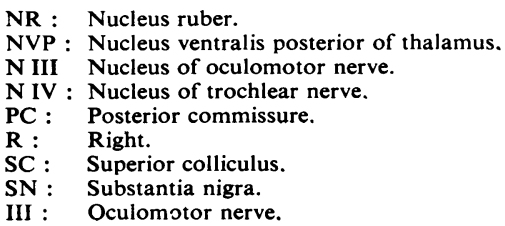

stained according to the methods of Weil, Bodian, and Davenport.

The Stereotactic Lesion.-The brain-stem lesion formed a sharply circumscribed and quite regular cavity (Fig. 2), which was filled with debris and macrophages and surrounded by a thin and sparse gliotic rim. In the rostro-caudal direction the lesion extended from the level of the posterior commissure to the rostral level of the trochlear nucleus (Fig. 3). The left medial lemniscus and spinothalamic tract apparently were completely destroyed. Furthermore, there was a considerable lesion of the mesencephalic reticular formation and the brachium of the inferior colliculus, and a small part of the red nucleus and superior colliculus was also damaged. Practically no lesion was found in the basal cerebral peduncle in spite of careful examination. Only a small tip in the lateralmost part of the peduncle, measuring 2-3 mm. ${ }^{2}$ in cross section, was damaged (Fig. 3). The fibres from this damaged area could be followed further caudally as a tiny and sharply localized gliotic segment, which disappeared among the lateralmost parts of the pontine nuclei (Fig. 3, dotted area). Sections stained by the Bodian, Weil, or Davenport methods did not reveal any degeneration of corticospinal fibres at the level of the lesion or further caudally.

Case 2.-The patient was a 50-year-old woman. In 1944, a sarcoma of the uterus was diagnosed, and the uterus was removed. After the operation she was free from symptoms until June, 1956, when she had severe pain in the left arm radiating to the three middle fingers. Radiographs showed multiple metastases in the lungs, but no bony metastases. She was admitted to the Neurosurgical Clinic in Lund on June 18, 1956. The pain, which was considered due to pressure on the brachial plexus from metastases in the supraclavicular fossa, could not be controlled by analgesics, and it was decided to do a right-sided stereotactic mesencephalic tractotomy.

Pre-operative Neurological Examination.-There were signs of peripheral nerve lesions in the left arm and hand, with pareses and hypoaesthesia corresponding to the lower cervical segments. The left triceps reflex was abolished. No other neurological abnormalities could be detected.

Operation.-The patient was operated on on July 9, 1956 , by the same technique as in the preceding case. The temperature in the centre of the lesion in this case reached $60^{\circ} \mathrm{C}$. Immediately after the operation she appeared to be analgesic on the left side. However, she was rather drowsy, and no detailed examination could be made.

Post-operative Course.-She recovered rapidly from the operation and was able to walk within one week. However, she then developed rapidly increasing dyspnoea and died 14 days after the operation. She had no pain in the left arm in the post-operative period.

Post-operative Neurological Examinations.-The information available about the post-operative neurological symptoms is somewhat less exact than in the preceding 


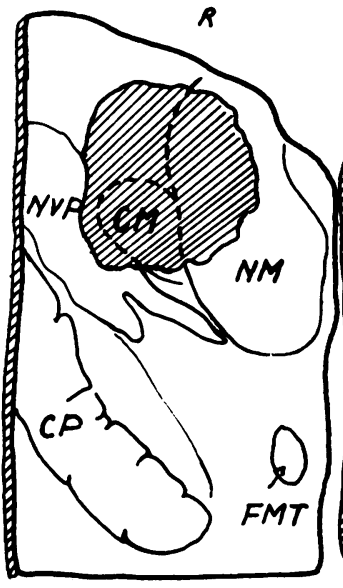

ROSTRAL

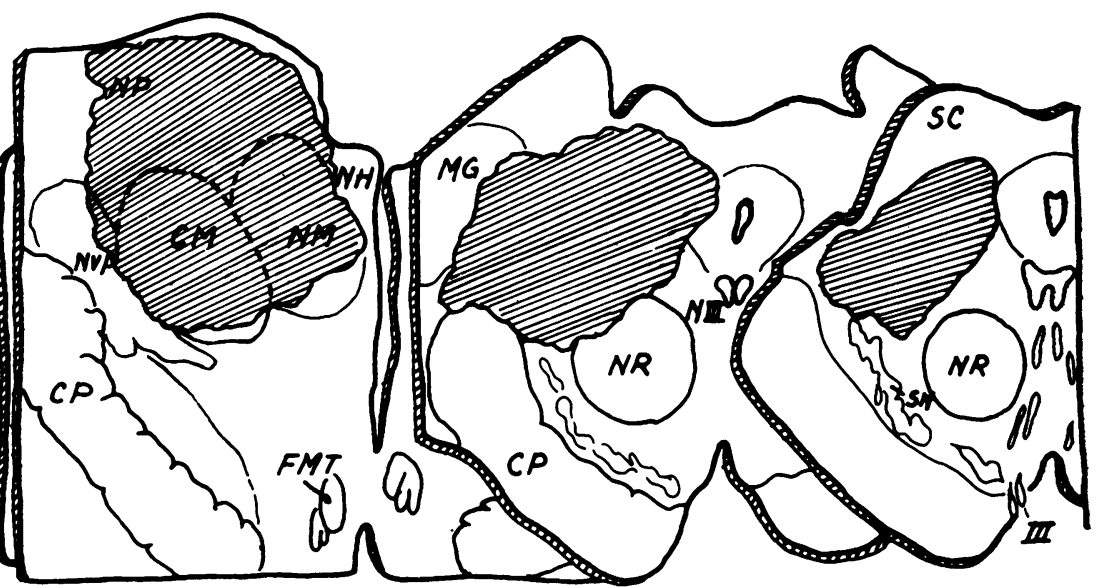

CAUOAL

FIG. 4.-Drawings made by means of a projection apparatus showing the extent of the lesion at various levels in Case 2.

BIC : Brachium of inferior colliculus.

CM : Centrum medianum.

CP : Cerebral peduncle (basis pedunculi).

FMT : Mamillothalamic fasciculus.

L: $\quad$ Left.

MG : Medial geniculate body.

NH : Habenular nuclei.

NM : Nucleus dorsomedialis of thalamus.

NP : Nuclei of pulvinar.
NR : Nucleus ruber.

NVP : Nucleus ventralis posterior of thalamus.

N III : Nucleus of oculomotor nerve.

N IV : Nucleus of trochlear nerve.

PC : Posterior commissure.

R : Right.

SC : Superior colliculus.

SN : Substantia nigra.

III : Oculomotor nerve. case. There was a left-sided hemi-hypalgesia to pinprick, which did not change during the post-operative course. The hypalgesia was more marked on the extremities than on the trunk and face, and apparently it was also more marked in the distal than proximal parts of the extremities. It is stated that there was a complete left-sided hemi-anaesthesia for temperature, touch, and muscle-joint sensibility. Nothing is mentioned about post-operative paraesthesiae and reflex findings. She apparently had no pareses.

Necropsy.-Widespread metastases from the sarcoma were found in the lungs, mediastinum, and heart. The brain and upper segments of the spinal cord were removed and fixed in $10 \%$ formalin. The entire brain was cut without finding evidence of metastases. The part of the brain containing the lesion was cut in transverse serial sections and treated in the same way as described for the preceding case.

The Stereotactic Lesion.-The lesion was considerably larger and more anteriorly placed than in the preceding case, extending in a rostro-caudal direction from the habenular nuclei to the caudal end of the medial geniculate body (Fig. 4). There was considerable damage to the medial parts of the thalamus. At more caudal levels the right lemniscus medialis and spinothalamic tract were probably completely destroyed. Also in this case the lesion was sharply delimited (Fig. 5).

Fig. 5.-Photomicrograph of Weil-stained section at the level of the lesion in Case 2. 
The central parts contained blood and necrotic tissue, surrounded by a rather dense zone of macrophages. The astroglial reaction was very slight. The greater extent of the lesion in this case was possibly in part due to an additional small haemorrhage.

\section{Discussion}

The Sensory System.-In spite of the large brainstem lesions in the two cases reported there was a considerable amount of sensibility left on the contralateral side of the body. When attempting to explain these findings, the first point of interest is whether parts of the medial lemniscus or spinothalamic tract might have escaped destruction. At caudal mesencephalic levels the medial lemniscus and spinothalamic tract cover a rather wide area on cross section. However, they converge somewhat towards the diencephalon, and according to the present knowledge about the localization of the tracts at these levels (Walker, 1940; Rasmussen and Peyton, 1941; Riley, 1943; Glees and Bailey, 1951), there can be little doubt that they were completely severed in both cases. Since most investigators studying the localization of the tracts have employed stains visualizing medullated fibres only, it might be objected that the finest fibres may be more dispersed than the medullated ones, thus having escaped destruction in the present cases. However, fibre analyses from cross sections of the spinal cord (Häggqvist, 1936), as well as experiences with cordotomies (White and Sweet, 1955), indicate that the finest fibres of the spinothalamic tracts probably run intermingled with the myelinated ones, and a further separation of fine and coarse spinothalamic fibres above the level of the spinal cord does not appear likely. Furthermore, considering the somatotopic localization in the spinothalamic tract and medial lemniscus, the finding of equal sensory loss in the arm and leg with relatively well preserved sensory perception on the trunk in both cases does not accord with the preservation of particular segments of the spinothalamic tract or medial lemniscus.

As will be known, anatomical as well as clinical findings indicate that some of the spinothalamic fibres ascend in the spinal cord without crossing to the opposite side (Foerster and Gagel, 1933; White and Sweet, 1955), while the fibres of the medial lemniscus appear to be entirely crossed (Rasmussen and Peyton, 1948). The question then arises whether the preserved sensory perception in the present cases may be explained by transmission of sensory impulses through ipsilateral spinothalamic fibres. If this is correct, the same degree of sensory loss would be expected following mesencephalic tractotomy and cordotomy in the spinal cord. However, complete section of the anterolateral quadrant of the cord is usually followed by longlasting contralateral analgesia, while some sensibility to pain frequently recurs shortly after mesencephalic tractotomy (White and Sweet, 1955). Furthermore, the distribution of the hypoaesthesia in the present cases, with relatively well preserved sensibility in the trunk, differs from the findings in cordotomies. It seems likely, therefore, that conduction through ipsilateral spinothalamic tract fibres cannot be the only explanation for the preserved sense perception in the cases reported.

These deliberations thus lead to the suggestion that in the brain-stem fibre systems other than the spinothalamic tract and medial lemniscus may be of importance for the perception and discrimination of sensory stimuli. From the first case described it appears that this applies to all cutaneous sensory qualities, while muscle-joint sensitivity, which was practically abolished, is probably almost entirely conveyed by the totally crossing medial lemniscus.

The number of direct spinothalamic fibres apparently is relatively small (Glees and Bailey, 1951), while numerous crossed and uncrossed spinoreticular, spino-tegmental, and spino-tectal fibres have been described (see Walker, 1940; Johnson, 1954; Rossi and Brodal, 1957; Bowsher, 1957). Similarly secondary trigemino-reticular fibres appear to be abundant (Ramón y Cajal, 1909; Scheibel, 1955; Nauta and Kuypers, 1958) Furthermore, reticulo-, tegmento-, and tectothalamic fibres have been described, particularly to the reticular and intralaminar thalamic nuclei (see Bucher and Bürgi, 1953; Johnson, 1953; Papez, 1956; Scheibel and Scheibel, 1958; Nauta and Kuypers, 1958). Admitting an extralemniscal component in the transmission of specific sensory impulses at brainstem levels, one cannot escape considering these plurisynaptic spino- and trigeminothalamic connexions as possible pathways. Some experimental support for this view as regards sensitivity to pain has recently been given by Melzack, Stotler, and Livingston (1958). These connexions have been considered of particular importance in the transmission of diffuse pain sensations related to the so-called thalamic syndrome (Hécaen, Talairach, David, and Dell, 1949; Bowsher, 1957). On account of the convergence of different sensory modalities on single reticular neurones and the diffuse cortical spread of ascending impulses through the intralaminar and reticular thalamic nuclei, it has been considered unlikely that this system participates in the transmission of impulses leading to perception, discrimination, and recognition of sensory stimuli (French, Verzeano, and 
Magoun, 1953). However, recent anatomical investigations have demonstrated reticulothalamic projections also to the specific thalamic nuclei (Scheibel and Scheibel, 1958; Nauta and Kuypers 1958), and convergence of impulses from different receptors on some of the reticular neurones scarcely excludes a more specific sensory transmission through the same system.

The contralateral facial and buccal sensitivity was particularly well preserved in the first case described, while no ipsilateral hypoaesthesia of the tongue was observed, as described by Walker (1942b). In this connexion the crossed and uncrossed dorsal trigeminothalamic tracts probably have to be considered as possible pathways of importance. It seems likely, although it cannot be proved, that the lesion in both cases also encroached upon the dorsal trigeminal tracts, while, of course, those on the opposite side remained intact. The information available about the anatomical and functional properties of these tracts is insufficient and in part contradictory (for reviews see Smyth, 1939; Walker, 1939; Russell, 1954; Berry, Anderson, and Brooks, 1956; Torvik, 1957). However, their possible role in specific sensory transmission cannot be entirely denied.

The distribution of the preserved sensitivity described in the present report (Fig. 1) is frequently encountered after cortical and thalamic lesions (Foerster, 1936; Környey, 1936). It has not been mentioned in other reports on mesencephalic tractotomy, but probably should be looked for in similar lesions. Such observations would substantiate the suggestion that in the caudal brainstem fibre systems other than the spinothalamic tracts and medial lemnisci are involved in the specific transmission of sensory impulses of different qualities.

The Reflex Changes.-Only the first case reported permits analysis of the possible influence of the brain-stem lesion on motor function and reflex mechanisms. This patient showed considerable increase of the contralateral tendon reflexes as well as extensor plantar response and weak abdominal reflexes during the entire post-operative course. Probably there was also some increase of the tendon reflexes of the ipsilateral leg. Damage to the corticospinal fibres in the brain-stem by the lesion itself or by post-operative oedema can probably be excluded as causes for these changes. Nor was there any evidence of cerebral metastases. However, since the spinal canal was not opened, the possibility of intraspinal metastases in the cervical region has to be taken into account. The refex changes appeared immediately after the operation, and in the following two and a half months there were no signs of progression in the neurological symptoms, except for some increase in tendon reflexes during the first weeks. Although not conclusive, this course would not be expected in intraspinal metastases from a rapidly growing tumour. The possibility thus remains that the "extrapyramidal" brain-stem lesion was responsible for the reflex changes observed, even if in current neurological thinking reflex changes of this kind have been considered almost pathognomonic of pyramidal tract lesions in man.

On the basis of animal experiments it has long been maintained that the spasticity and hyperreflexia following lesions of the internal capsule are chiefly caused by damage of extrapyramidal projection fibres (see Tower, 1949). According to Mettler (1944) and Mettler and Zimmerman (1943), the latter fibres probably cross the midline at the level of the rostral pole of the red nucleus. Conclusive human observations with regard to these problems are few, but several investigators stress the importance of pyramidal tract lesions in spasticity and hyperreflexia in man (Walshe, 1947; Lassek, 1954). However, the recent observations by Bucy (1957) that section of the corticospinal fibres at the level of the mesencephalon may give rise to only slight pareses and very little spasticity and hyperreflexia, strongly suggest an important extrapyramidal component in the production of spastic pareses also in man. From the observations of Mettler (1944) and Mettler and Zimmerman (1943), it does not appear unlikely that in the present case the lesion at the level of the red nucleus might provoke bilaterally increased tendon reflexes.

There is little doubt that pyramidal tract lesions in man and anthropoid apes usually give rise to extensor plantar response and weak or abolished abdominal reflexes (Fulton and Keller, 1932; Tower, 1949; Lassek, 1954). Less is known of whether these reflex changes may also occur following other lesions of the central nervous system. According to Lassek (1954), the Babinski sign may probably occur in man without pyramidal tract affection, and Nathan and Smith (1955) in cordotomy material found extensor plantar responses in several cases without lesions of the pyramidal tract, as well as normal plantar reflexes in cases with definite damage to corticospinal fibres. Corresponding observations apparently have not been made with regard to abdominal reflexes. However, according to the findings in the present report, it seems possible that both plantar and abdominal reflexes may be influenced by brain-stem lesions leaving the pyramidal tract intact. 


\section{Summary}

Two cases with intractable pain are reported in which unilateral stereotactic brain-stem lesions were made in order to destroy the spinothalamic tracts. Both patients suffered from advanced cancer with metastases. One of the patients, who pre-operatively had severe pain in the right arm and chest, lived for two and a half months after the operation. During the last weeks there was some recurrence of the pain in the chest, but not in the arm. The other patient died from pulmonary metastases 14 days after the operation. She was relieved from the pain during the post-operative course.

In both cases some sensitivity was preserved on the contralateral side of the body, particularly on the trunk and face, in spite of complete destruction of the spinothalamic tract and medial lemniscus. It is suggested that transmission of sensory impulses through fibre systems other than the spinothalamic tracts and medial lemnisci in the brain-stem may be responsible for the preserved sensibility.

In the first case the tendon reflexes, extensor plantar response, and weak abdominal reflexes were increased, on the contralateral side of the lesion and probably also the tendon reflexes in the homolateral leg were somewhat exaggerated. Postmortem examination of the brain-stem showed no involvement of the corticospinal fibres. The findings indicate that tendon reflexes as well as abdominal and plantar reflexes may be influenced by lesions of extrapyramidal fibre systems.

\section{REFERENCES}

Berry, C. M., Anderson, F. D., and Brooks, D. C. (1956). J. Neurophysiol., 19, 144.

Bowsher, D. (1957). Brain, 80, 606.

Bucher, V. M., and Bürgi, S. M. (1953). J. comp. Neurol., 99, 415.

Bucy, P. C. (1957). Brain, 80, 376
David, M., Talairach, J., and Hécaen, H. (1947). Sem. Hóp. Paris. $23,1651$.

Dogliotti, A. M. (1938). Anésth. et Analg., 17, 143.

Foerster, O. (1936). In Bumke, O. and Foerster, O. Handbuch der Neurologie, Vol. 6, p. 358. Springer, Berlin.

-_. and Gagel, O. (1933). Z. ges. Neurol. Psychiat., 144. 313.

French, J. D., Verzeano, M., and Magoun, H. W. (1953). A. M. A. Arch. Neurol. Psvchiat., 69, 505.

Fulton, J. F., and Kelier, A. D. (1932). The Sign of Babinski. A Study of the Evolution of Cortical Dominance in Primates. Study of the Evolution of Cortical Dom

Glees, P.. and Bailey, R. A. (1951). Mschr. Psychiat. Neurol.. 122. 129.

Guiot, G., and Forjaz, S. (1947). Rev. neurol., 79, 733.

Häggqvist, G. (1936). Z. mikr.-anat. Forsch., 39, 1.

Hécaen, H., Talairach, J., David, M., and Dell, M. B. (1949). Rev. neurol., 81, 917.

Johnson, F. H. (1953) Anat. Rec., 115, 327.

Johnson, F. H. Ibid., 118, 316 .

Környey, St. (1936). In Bumke, O., and Foerster, O. Handbuch der Neurologie, Vol. 5, p. 445. 'Springer, Berlin.

Lassek, A. M. (1954). The Pyramidal Tract. Charles C. Thomas. Springfield, Illinois. Amer. Lect. Ser. No, 233.

Leksell, L. (1957). In Olivecrona, H., and Tönnis, W. : Handbuch der Neurochirugie, Vol. 6, p. 178 . Springer, Berlin.

Melzack, R., Stotler, W. A., and Livingston, W. K. (1958). J. Neir.. physiol., 21, 353.

Mettler, F. A.'(1944). J. comp. Neurol., 80, 69.

- - , and Zimmerman, F. T. (1943). Ibid., 78, 113

Nathan, P. W., and Smith, M. C. (1955). J. Neurol. Neurosurg. Psychiat., 18, 250.

Nauta, W. J. H., and Kuypers, H. G. J. M. (1958). In Reticular Formation of the Brain, p. 3. [Henry Ford Hospital International Symposium]. Little, Brown and Co., Boston.

Papez, J. W. (1956). Electroenceph. clin. Neurophysiol., 8, 117.

Ramón y Cajal, S. (1909). Histologie du Systéme Nerveux de l'Homme et des Vertébrés, Vol. 1. Maloine, Paris.

Rasmussen, A. T., and Peyton, W. T. (1941). Surgery, 10, 699.

- - - (1948). J. comp. Neurol., 88, 411.

Riley, H. A. (1943). An Atlas of the Basal Ganglia, Brain Stem and Spinal Cord. Williams and Wilkins, Baltimore.

Rossi, G. F.. and Brodal, A. (1957). A.M.A. Arch. Neurol. Psychiat. $78,439$.

Russell, G. V. (1954). J. comp. Neurol., 101, 237.

Scheibel, A. B. (1955). Anat. Rec., 121, 361.

Scheibel, M. E., and Scheibel, A. B. (1958). In Reticular Formation of the Brain, p. 31. Henry Ford Hospital, Detroit, Michigan.

Schwartz, H. G. (1950). Surg. Clin. N. Amer., 30, 1379.

Siöquist, O. (1949). IVe Congr. Neurol., 1, 119.

Sjöquist, O. (1949). IVe Congr. Neurot.

Torvik, A. (1957). Amer. J. Anat., 100, 1.

Tower, S. (1949). In Bucy, P. C. The Precentral Motor Cortex, 2nd ed., p. 149. University of Illinois Press, Urbana.

Walker, A. E. (1939). J. comp. Neurol., 71, 59.

(1940). Arch. Neurol. Psychiat. (Chicago), 43, 284.

(1942a). Arch. Surg. (Chicago), 44, 953.

- (1942a). Arch. Surg. (Chicago), 44, 953.

- (1942b). Arch. Neurol. Psychiat. (Chicago), 48, 865. 1950). J. Lancet, 70, 279. Cited from White and Sweet (1955).

Walshe, F. M. R. (1947). Brain, 70, 329.

White, J. C., and Sweet, W. H. (1955). Pain, Its Mechanisms and Neurosurgical Control. Charles C. Thomas, Springfield, Illinois. 